\title{
Gal4 turnover and transcription activation
}

\author{
Arising from: K. Nalley, S. A. Johnston \& T. Kodadek Nature 442, 1054-1057 (2006)
}

Growing evidence supports the notion that proteasome-mediated destruction of transcriptional activators can be intimately coupled to their function ${ }^{1,2}$. Recently, Nalley et al. ${ }^{3}$ challenged this view by reporting that the prototypical yeast activator Gal4 does not dynamically associate with chromatin, but rather 'locks in' to stable promoter complexes that are resistant to competition. Here we present evidence that the assay used to reach this conclusion is unsuitable, and that promoter-bound, active Gal4 is indeed susceptible to competition in vivo. Our data challenge the key evidence that Nalley et al. ${ }^{3}$ used to reach their conclusion, and indicate that Gal4 functions in vivo within the context of dynamic promoter complexes.

Studies by several groups, including ours ${ }^{1,2,4-6}$, have reported an intimate connection between the activity of transcriptional activators such as Gal4 and Gcn4 and their destruction by ubiquitin-mediated proteolysis. This intimate connection is difficult to reconcile with the conclusion by Nalley et al. ${ }^{3}$ that proteolytic turnover of Gal4 is not coupled to its function. This conclusion is based on the result of chromatin immunoprecipitation (ChIP) experiments showing that endogenous, active, Gal4 cannot be competed from the GAL1/10 promoter by induction of a protein with the same DNA-binding specificity ('competitor'). In this case, the competitor contains the hormone-binding domain of the oestrogen receptor (ER), which allows its DNA-binding activity to be rapidly induced by the treatment of yeast with $\beta$-oestradiol. Yeast cultures expressing both the competitor and endogenous Gal4 are treated with $\beta$-oestradiol, and ChIP analysis is used to monitor the binding of the two proteins to the GAL1/10 promoter.

We obtained reagents from the Kodadek laboratory and repeated their experiments. In the course of performing an additional control that was not included in their Nature paper, we observed that, in the absence of any competitor, $\beta$-oestradiol induced an up to fourfold increase in the levels of Gal4 that associated with the GAL1/10 promoter (Fig. 1a, blue line). The unexpected ability of $\beta$-oestradiol to induce binding of endogenous Gal4 makes the competition assay difficult to interpret, as the compound is simultaneously inducing both the competitor and the species being competed.
To explore this issue further, we repeated the experiment using a different ER ligand, 4-hydroxytamoxifen (4HT). In the absence of competitor, 4HT had little effect on the association of endogenous Gal4 with its cognate promoter (Fig. 1a, red line). Consistent with the different effects of these two ligands on basal association of Gal4 with chromatin, the two compounds gave very different results in the presence of competitor (Fig. 1b). As Nalley et al. published ${ }^{3}$, the addition of $\beta$-oestradiol to yeast expressing the competitor protein resulted in little if any reduction in the levels of endogenous Gal4 at the GAL1/10 promoter, creating the impression that most promoter-bound Gal4 resisted competition (Fig. 1b, blue line). In the presence of $4 \mathrm{HT}$, however, the opposite result was obtained, and $\sim 75 \%$ of endogenous Gal 4 was competed from the chromatin within $15 \mathrm{~min}$ of the ligand addition (Fig. 1b, red line). Notably, the loss of Gal4-chromatin association was accompanied by loading of the competitor onto the GAL1/10 promoter (Fig. 1c), consistent with the notion that the $4 \mathrm{HT}$-activated competitor can displace endogenous Gal4 from the promoter. Although the competitor protein associated with the GAL1/10 promoter with apparently slower kinetics than endogenous Gal4 dissociated (compare red line in Fig. 1b with pink line in Fig. 1c), it is worth noting that endogenous Gal4 can bind cooperatively to several sites in vivo ${ }^{7}$. There are four Gal4-binding sites in the GAL1/10 promoter. Thus, a single competitor bound to one of the sites could have the effect of destabilizing multiple Gal4-promoter complexes, leading to efficient displacement of endogenous Gal4 at substoichiometric levels of competitor.

On the basis of our observations, we propose that the recalcitrance of Gal4-promoter complexes originally reported by Nalley et al. ${ }^{3}$ is an artefact of using $\beta$-oestradiol to stimulate the competitor. Activating the competitor with $4 \mathrm{HT}$ (Fig. 1b), or normalizing the $\beta$-oestradiol signal to the important 'no competitor' control (Fig. 1d), shows that Gal4 can indeed be rapidly displaced from promoter DNA in vivo. Their conclusion that Gal4-promoter complexes lock in and have long half-lives under activating conditions is thus unsustainable.
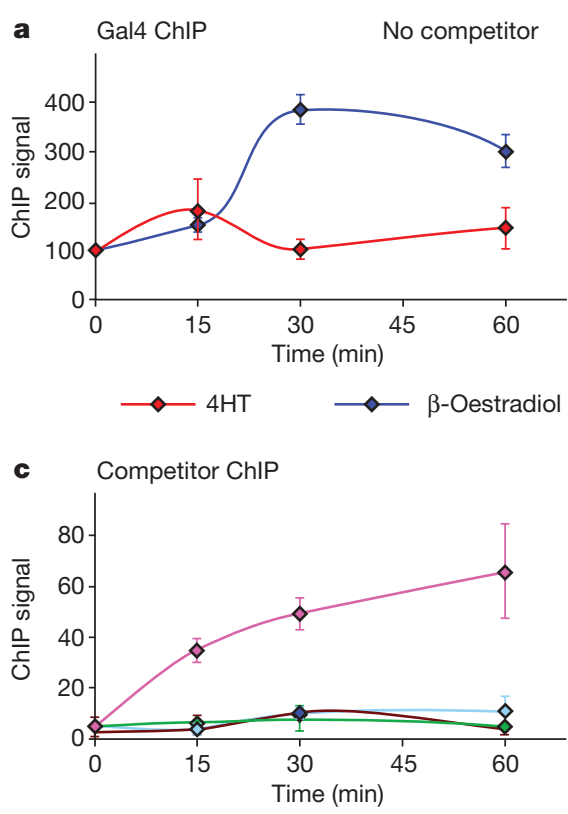

$\diamond 4 \mathrm{HT}+$ competitor $\diamond$ Ethanol + competitor
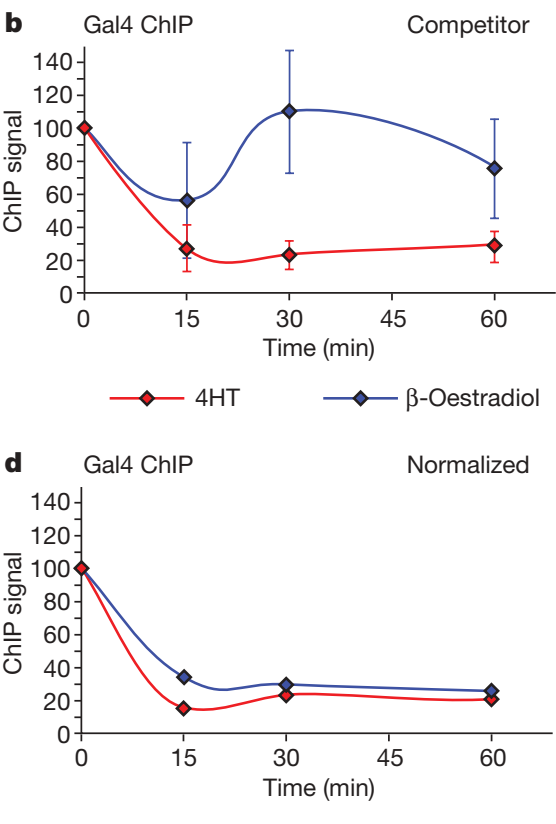

$\sim 4 \mathrm{HT}$ - competitor $\sim$ Ethanol - competitor
Figure 1 Activation of a Gal4 competitor with $\beta$-oestradiol versus 4HT. a, Wild-type yeast were induced with $2 \%$ galactose for $60 \mathrm{~min}$ and $\beta$-oestradiol or $4 \mathrm{HT}$ was added. At indicated times, the occupancy of endogenous Gal4 on the GAL1/10 promoter was determined by ChIP. ChIP signal is normalized to that at time zero. b, As in a, except that experiment was performed in yeast expressing the Myc-G4-ER-VP16 competitor (supplied by T. Kodadek ${ }^{3}$ ). c, As in the $4 \mathrm{HT}$ experiment in b, except that ChIP was used to monitor association of the

Myc-G4-ER-VP16 competitor with the GAL1/10 promoter. The corresponding non-competitor controls are also shown. To calculate the percentage binding in this case, ChIP signals were normalized to those from a Myc-G4-ER-VP16 ChIP (60-min time point) performed in the absence of endogenous Gal4, which corresponds to the total amount of competitor that can bind in this assay. $\mathbf{d}$, ChIP signals from $\beta$-oestradiol or 4 HT experiments in $b$ normalized to the relevant 'no competitor' control in a. Error bars are s.e.m. $(n=3)$. 


\section{METHODS}

Yeast (BY4741) with or without competitor (Myc-G4-ER-VP16) ${ }^{3}$ were grown in complete synthetic medium (CSM) (2\% raffinose) and Gal4 was induced by transferring yeast to media containing $2 \%$ galactose for $1 \mathrm{~h}$. Yeast were then treated with $1 \mu \mathrm{M} 17-\beta$ oestradiol (Sigma) or $100 \mu \mathrm{M}$ 4-hydroxytamoxifen (Sigma) for the indicated times. ChIP was preformed ${ }^{5}$ using either the Gal4TA C-10 (anti-GAL4; Santa Cruz) or AB1 (anti-Myc; Calbiochem) antibodies. DNA enrichment was calculated as described ${ }^{8}$ using ACT1 as the reference locus. Galen A. Collins ${ }^{1,2 \dagger}$, J. Russell Lipford ${ }^{3 \dagger}$, Raymond J. Deshaies ${ }^{3}$ \& William P. Tansey ${ }^{2 \dagger}$

${ }^{1}$ Watson School of Biological Sciences, 1 Bungtown Road, Cold Spring Harbor, New York 11724, USA.

${ }^{2}$ Cold Spring Harbor Laboratory, 1 Bungtown Road, Cold Spring Harbor, New York 11724, USA.

e-mail: william.p.tansey@vanderbilt.edu

${ }^{3}$ Caltech, Division of Biology, 1200 East California Boulevard, Pasadena, California 91125, USA.

†Present addresses: Vanderbilt University Medical Centre, 465 21st

Avenue South, Nashville, Tennessee 37232, USA (G.A.C. and W.P.T.);
Amgen, 1 Amgen Center Drive, Thousand Oaks, California 91320-1799, USA (J.R.L.).

\section{Received 20 February; accepted 13 August 2009.}

1. Lipford, J. R. \& Deshaies, R. J. Diverse roles for ubiquitin-dependent proteolysis in transcriptional activation. Nature Cell Biol. 5, 845-850 (2003).

2. Muratani, M. \& Tansey, W. P. How the ubiquitin-proteasome system controls transcription. Nature Rev. Mol. Cell Biol. 4, 192-201 (2003).

3. Nalley, K., Johnston, S. A. \& Kodadek, T. Proteolytic turnover of the Gal4 transcription factor is not required for function in vivo. Nature 442, 1054-1057 (2006).

4. Lipford, J. R., Smith, G. T., Chi, Y. \& Deshaies, R. J. A putative stimulatory role for activator turnover in gene expression. Nature 438, 113-116 (2005).

5. Muratani, M., Kung, C., Shokat, K. M. \& Tansey, W. P. The F box protein Dsg1/Mdm30 is a transcriptional coactivator that stimulates Gal4 turnover and cotranscriptional mRNA processing. Cell 120, 887-899 (2005).

6. Salghetti, S. E., Muratani, M., Wijnen, H., Futcher, B. \& Tansey, W. P. Functional overlap of sequences that activate transcription and signal ubiquitin-mediated proteolysis. Proc. Natl Acad. Sci. USA 97, 3118-3123 (2000).

7. Giniger, E. \& Ptashne, M. Cooperative DNA binding of the yeast transcriptional activator GAL4. Proc. Natl Acad. Sci. USA 85, 382-386 (1988).

8. Ezhkova, E. \& Tansey, W. P. Chromatin immunoprecipitation to study protein-DNA interactions in budding yeast. Methods Mol. Biol. 313, 225-244 (2006).

doi:10.1038/nature08406

\section{Nalley et al. reply}

\section{Replying to: G. A. Collins, J. R. Lipford, R. J. Deshaies \& W. P. Tansey Nature 461, doi:10.1038/nature08406 (2009)}

Proteasome-mediated turnover of some ${ }^{1,2}$, but clearly not all ${ }^{3,4}$, transcriptional activators is important for their activity. To facilitate the analysis of activator-promoter complex lifetime in vivo, a parameter relevant to this issue, we developed a competition chromatin immunoprecipitation (ChIP) assay in which binding of a native transactivator to its cognate promoters is challenged by a ligand-activated competitor protein with the same DNA-binding specificity. We applied this technique to the yeast Gal4 system ${ }^{5}$ and concluded that under noninducing conditions (raffinose media) Gal4-promoter complexes exchange rapidly, but under inducing conditions (galactose media) the activator-promoter complexes are long-lived. Collins et al. ${ }^{6}$ report that, surprisingly, the addition of oestradiol to yeast lacking Myc-G4ER-VP16 increased the amount of DNA co-immunoprecipitated with native Gal4.

This is a control we had not done, but have subsequently repeated and agree that this is the case (S.A.J. wishes to note that he had requested this control and it erroneously was not done). We thank Collins et al. ${ }^{6}$ for pointing out this omission. They go on to show that inducing competitor protein activity with 4-hydroxytamoxifen (4HT) results in a significant loss in the intensity of the ChIP signal owing to native Gal4, but that this ligand does not affect the intensity of these ChIP signals in the absence of competitor. They also show significant association of the competitor protein with the promoter, although with a different time course than Gal4 dissociation. We agree that these data indicate that a large fraction of Gal4-promoter complexes are kinetically labile in vivo under these (4HT-containing media) conditions. It is important to note that this odd effect of steroid is not a general problem in the application of this technology to the measurement of other activator-promoter half-lives ${ }^{4}$.

However, our ChIP data tracking association of the competitor protein do not support the conclusion of a rapidly exchanging Gal4-DNA complex in vivo in the presence of $\beta$-oestradiol rather than 4 HT. There is no indication that these data are compromised by unanticipated effects of $\beta$-oestradiol. Under inducing conditions, much lower levels of association of the competitor protein with $G A L$ promoters were observed when Gal4 was present than in 4 gal4 cells when $\beta$-oestradiol was used to trigger the competition. These data argue for the presence of a stable, functional Gal4-promoter complex in the presence of galactose and under the particular conditions used in our study ${ }^{5}$. It may be that the stability of Gal4-promoter complexes are somehow affected by steroid receptor ligands, which would explain the different results observed by ourselves and Collins et al. ${ }^{6}$ for the association of the competitor protein in our respective experiments.

\section{Kip Nalley ${ }^{1} \uparrow$, Stephen Albert Johnston ${ }^{1} \dagger \&$ Thomas Kodadek ${ }^{1} \dagger$}

${ }^{1}$ Department of Internal Medicine, UT Southwestern Medical Center, 5323 Harry Hines Boulevard, Dallas, Texas 75390-9185, USA.

Email: kodadek@scripps.edu

†Present addresses: Laboratory of Receptor Biology and Gene

Expression, National Cancer Institute, Building 41, Room B602, 41 Library Drive MSC 5055, Bethesda, Maryland 20892-5055, USA (K.N.); Center for Innovations in Medicine, Biodesign Institute, Arizona State University, Tempe, Arizona 85287-5001, USA (S.A.J.); Departments of Chemistry \& Cancer Biology, Scripps Research Institute, Scripps Florida, 130 Scripps Way, Jupiter, Florida 33458, USA (T.K.).

1. Reid, G. et al. Cyclic, proteasome-mediated turnover of unliganded and liganded ER $\alpha$ on responsive promoters is an integral feature of estrogen signaling. Mol. Cell 11, 695-707 (2003).

2. Lipford, J. R. et al. A putative stimulatory role for activator turnover in gene expression. Nature 438, 113-116 (2005).

3. Yao, J. et al. Dynamics of heat shock factor association with native gene loci in living cells. Nature 442, 1050-1053 (2006).

4. Yu, P. \& Kodadek, T. Dynamics of the hypoxia-inducible factor-1-vascular endothelial growth factor promoter complex. J. Biol. Chem. 282, 35035-35045 (2007).

5. Nalley, K., Johnston, S. A. \& Kodadek, T. Proteolytic turnover of the Gal4 transcription factor is not required for function in vivo. Nature 442, 1054-1057 (2006).

6. Collins, G. A., Lipford, J. R., Deshaies, R. J. \& Tansey, W. P. Gal4 turnover and transcription activation. Nature 461, doi:10.1038/nature08406 (2009).

doi:10.1038/nature08407 